\title{
“SOBRE AS SOMAS DAS SÉRIES DE RECÍPROCOS”, DE L. EULER
}

\author{
Frederico J. A. Lopes \\ Universidade Federal de Mato Grosso - UFMT - Brasil
}

(aceito para publicação em maio de 2021)

\section{Resumo}

Esta é uma tradução do artigo De summis serierum reciprocarum (Sobre a soma das séries de recíprocos), de Leonhard Euler (1707-1783), em que ele resolve o famoso problema de Basileia.

Palavras-chave: problema de Basileia, história, Euler.

\section{[ON THE SUMS OF SERIES OF RECIPROCALS]}

\begin{abstract}
This is a translation of the paper De summis serierum reciprocarum (On the sums of series of reciprocals), by Leonhard Euler (1707-1783), where he solves the famous Basel Problem.
\end{abstract}

Keywords: Basel problem, history, Euler.

\section{Introdução}

O artigo De summis serierum reciprocarum (Sobre as somas das séries de recíprocos) trata da solução que L. Euler (1707-1783) deu ao problema conhecido como o problema de Basileia: encontrar a soma da série $1+\frac{1}{4}+\frac{1}{9}+\frac{1}{16}+\frac{1}{25}+$ etc. na forma de uma expressão numérica fechada, envolvendo algumas constantes conhecidas e outras poucas operações aritméticas. 
Originalmente proposto por Pietro Mengoli (1625-1686), esse problema se mantinha sem solução no tempo de Euler. $O$ valor aproximado de 1,6449340668482264364..., como Euler nos apresenta com precisão espantosa, era conhecido, mas ninguém sabia ainda como encontrá-lo a não ser pelo processo insatisfatório de somar cada termo. Sua resolução foi tentada pelas gerações anteriores de matemáticos, notadamente por Johann Bernoulli (1667-1748), o mentor de Euler.

Quando Euler entra em cena, o problema acaba resolvido em apenas onze parágrafos, e ele encontra, como solução, o valor exato de $\frac{\pi^{2}}{6}$. Mas ele continua por mais oito parágrafos derivando consequências do método que o levou à solução. E, como é padrão em seus escritos, Euler se vangloria mais do seu método do que de ter resolvido o próprio problema, e é pelo seu método, a nosso ver, que este artigo merece atenção.

Somos da opinião, compartilhada por muitos, que a leitura das obras originais costuma ser mais instrutiva e formadora do que a leitura de um bom livro didático. É nesse sentido que realizamos essa tradução, aguardando também críticas e alternativas tradutórias que possam engrandecer e facilitar a leitura desse trabalho.

\section{Bibliografia}

EULER, Leonhard. De summis serierum reciprocarum. Commentarii academiae scientiarum Petropolitanae, Vol. 7 (1734/35), 1740, pp. 123-134. Fac-símile em <https://www.biodiversitylibrary.org/item/38582>. Acesso: 26 de maio de 2021.

SANDIFER, Edward. Estimating the Basel Problem. MAA Online, dezembro de 2003. Disponível em <http://eulerarchive.maa.org/hedi/>. Acesso: 03 de setembro de 2020.

\section{Frederico José Andries Lopes}

Departamento de Matemática - UFMT - Campus de Cuiabá - Brasil

E-mail: contato@fredlopes.com.br 


\section{A tradução}

\section{Sobre as somas das séries de recíprocos}

\section{$\S .1$.}

As séries recíprocas de potências de números naturais já foram de tal maneira examinadas e investigadas que parece bem pouco provável que se possa encontrar algo de novo sobre elas. Pois quase todos os que refletiram sobre somas de séries inquiriram também sobre as somas destas séries e, entretanto, não as puderam exprimir satisfatoriamente por nenhum método. Eu também, tendo apresentado vários métodos de somá-las, investiguei frequentemente essas séries com diligência e, no entanto, não consegui nada senão definir aproximadamente suas somas exatas ou reduzi-las a quadraturas de curvas principalmente transcendentes, o que já apresentei em uma dissertação publicada há pouco também na precedente. Falo aqui, no entanto, de séries de frações cujos numeradores são 1 e os denominadores são quadrados, cubos ou outras potências de números naturais, por exemplo $1+\frac{1}{4}+\frac{1}{9}+\frac{1}{16}+\frac{1}{25}+$ etc. ou também $1+\frac{1}{8}+\frac{1}{27}+\frac{1}{64}+$ etc. e semelhantes de potências superiores cujo termo geral tem a forma $\frac{1}{x^{n}}$.

§.2. Recentemente fui conduzido, completamente de surpresa, a uma elegante expressão da soma da série $1+\frac{1}{4}+\frac{1}{9}+\frac{1}{16}+\frac{1}{25}+$ etc, que depende da quadratura do círculo de tal maneira que, se a verdadeira soma da série for obtida, daí imediatamente se segue a quadratura do círculo. Pois descobri que o sêxtuplo da soma desta série é igual ao quadrado da periferia ${ }^{1}$ do círculo cujo diâmetro é 1 ; em outras palavras, posta a soma da série $=s$, então a razão da periferia para o diâmetro será de $\sqrt{6 s}$ para 1. Mostrei recentemente, porém, que a soma dessa série é aproximadamente 1,6449340668482264364, número que, extraída a raiz quadrada do seu sêxtuplo, dará o número 3,141592653589793238, que exprime a periferia do círculo de diâmetro 1. Em seguida percebi, da mesma maneira como cheguei a essa soma, que a soma da série $1+\frac{1}{16}+\frac{1}{81}+\frac{1}{256}+\frac{1}{625}+$ etc também depende da quadratura do círculo. De fato, essa soma, multiplicada por 90, dá o biquadrado ${ }^{2}$ da periferia do círculo de diâmetro 1. E, da mesma maneira, pude determinar também as somas das séries seguintes nas quais os expoentes das potências são números pares.

§.3. Para mostrar, portanto, como cheguei a essa conclusão, farei uma exposição clara do método que utilizei. No círculo descrito AMBNA de centro $\mathrm{C}$ e raio $\mathrm{AC}$ ou $\mathrm{BC}=1$, tomei um arco qualquer AM, cujo seno é MP e o cosseno é CP.

\footnotetext{
${ }^{1}$ Euler quer dizer circunferência. Assim também "semiperiferia” quer dizer semicircunferência.

${ }^{2}$ Biquadrado indica o quadrado do quadrado, ou seja, a quarta potência.
} 


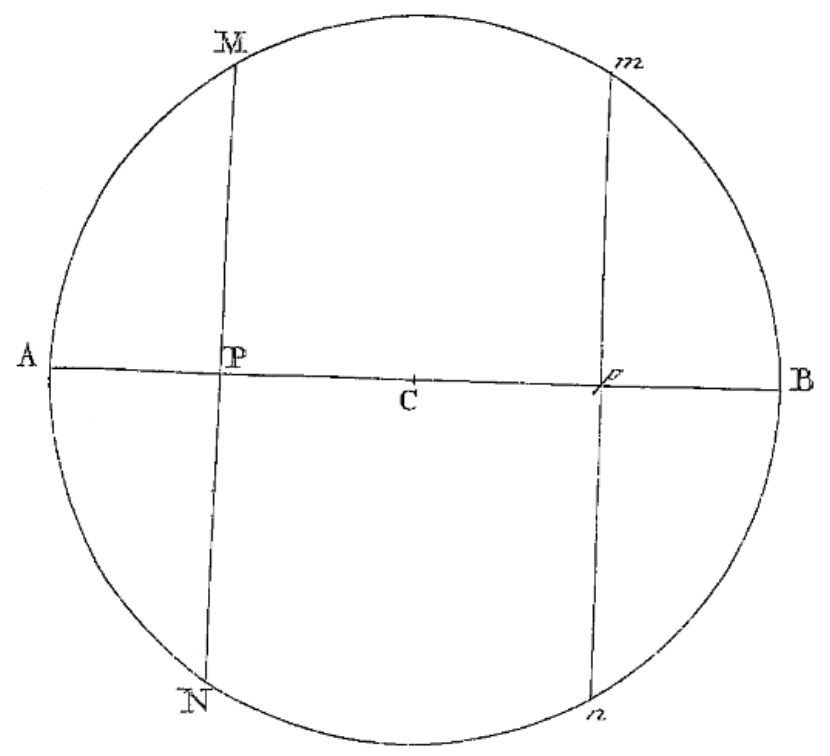

Posto agora que o arco $\mathrm{AM}=s$, o seno $\mathrm{PM}=y$ e o cosseno $\mathrm{CP}=x$, por um método já bem conhecido, tanto o seno $y$ quanto o cosseno $x$ podem ser definidos por séries a partir do arco dado $s$, como se vê em toda parte: $\mathrm{y}=\mathrm{s}-\frac{s^{3}}{1.2 .3}+\frac{s^{5}}{1.2 .3 .4 .5}-\frac{s^{7}}{1.2 .3 .4 .5 .6 .7}+$ etc e $\mathrm{x}=1-\frac{s^{2}}{1.2}+\frac{s^{4}}{1.2 .3 .4}-\frac{s^{6}}{1.2 .3 .4 .5 .6}+$ etc. A partir da consideração destas séries, cheguei às somas das séries de recíprocos referidas mais acima; em verdade, ambas equações servem quase ao mesmo propósito, e por isso basta que eu trate de uma só, o que passo a fazer a seguir.

§.4. A primeira equação $\mathrm{y}=\mathrm{s}-\frac{s^{3}}{1.2 .3}+\frac{s^{5}}{1.2 .3 .4 .5}-\frac{s^{7}}{1.2 .3 .4 .5 .6 .7}+$ etc exprime uma relação entre o arco e o seno. Com ela, tanto o seno poderá ser determinado a partir de um arco dado, quanto o arco a partir de um seno dado. Vou considerar então o seno y como dado e vou investigar como encontrar o arco $s$ a partir de $y$. Mas antes de tudo é preciso observar que a cada seno y correspondem inumeráveis arcos e, portanto, que a equação proposta deve fornecer esses inumeráveis arcos. De fato, se nessa equação $s$ é visto como uma incógnita, ela tem infinitas dimensões, e por isso não é de se espantar que esta equação contenha inúmeros fatores simples, qualquer um dos quais, se feito igual a zero, deve dar um valor conveniente para $s$.

§.5. Se todos os fatores dessa equação fossem conhecidos, também todas as raízes ou valores de s seriam conhecidos; por sua vez, se todos os valores de $s$ pudessem ser atribuídos, então todos os fatores dessa equação também seriam encontrados. Mas para que 
eu possa tratar melhor tanto das raízes quanto dos fatores, transformo a equação proposta nesta forma $0=1-\frac{s}{y}+\frac{s^{3}}{1.2 \cdot 3 \cdot y}-\frac{s^{5}}{1 \cdot 2 \cdot 3 \cdot 4 \cdot 5 \cdot y}+$ etc. Agora, se todas as raízes desta equação ou todos os arcos que têm o mesmo seno $y$ forem $A, B, C, D, E$ etc., então os fatores também serão todas essas quantidades $1-\frac{s}{A}, 1-\frac{s}{B}, 1-\frac{s}{C}, 1-\frac{s}{D}$ etc. Daí será $1-\frac{s}{y}+\frac{s^{3}}{1.2 \cdot 3 \cdot y}-\frac{s^{5}}{1 \cdot 2 \cdot 3 \cdot 4 \cdot 5 \cdot y}+$ etc. $=\left(1-\frac{s}{A}\right)\left(1-\frac{s}{B}\right)\left(1-\frac{s}{C}\right)\left(1-\frac{s}{D}\right)$ etc.

§.6. Da natureza e da resolução das equações, o coeficiente $\frac{1}{y}$ do termo no qual se encontra $s$ é igual à soma de todos os coeficientes de $s$ nos fatores, ou seja, $\frac{1}{y}=\frac{1}{A}+\frac{1}{B}+\frac{1}{C}+\frac{1}{D}+$ etc. E também o coeficiente de $s^{2}$, que é $=0$, e por isso esse termo está ausente na equação, é igual à multiplicação de fatores da série $\frac{1}{A}, \frac{1}{B}, \frac{1}{C}, \frac{1}{D}$ etc. tomados dois a dois. E daí que $\frac{1}{1.2 .3 \cdot y}$ será igual à multiplicação dos fatores da série $\frac{1}{A}$, $\frac{1}{B}, \frac{1}{C}, \frac{1}{D}$ etc. tomados três a três ${ }^{3}$. De maneira semelhante, será $=0$ a multiplicação de fatores da mesma série tomados quatro a quatro, e $\frac{1}{1.2 .3 .4 .5 . y}=$ à multiplicação de fatores da mesma série tomados cinco a cinco, e assim por diante.

§.7. Posto agora o arco mínimo $\mathrm{AM}=\mathrm{A}$, cujo seno é $\mathrm{PM}=y$, e a semiperiferia do círculo $=$ p, serão A, p - A, $2 p+A, 3 p-A, 4 p+A, 5 p-A, 6 p+A$ etc., e também -p - A, $-2 p+A$, $-3 p-A,-4 p+A,-5 p-A$, etc. todos os arcos cujo seno é também $y$. Portanto, tomada a série anterior $\frac{1}{A}, \frac{1}{B}, \frac{1}{C}, \frac{1}{D}$, etc, ela se transforma em $\frac{1}{A}, \frac{1}{p-A}, \frac{1}{-p-A}, \frac{1}{2 \mathrm{p}+\mathrm{A}}$, $\frac{1}{-2 \mathrm{p}^{+\mathrm{A}}}, \frac{1}{3 p-A}, \frac{1}{-3 p-A}, \frac{1}{4 \mathrm{p}^{+\mathrm{A}}}, \frac{1}{-4 \mathrm{p}+\mathrm{A}}$, etc. Daí que a soma de todos estes termos é $=\frac{1}{y}$, mas a soma dos fatores desta série [multiplicados] dois a dois é igual a 0 ; a soma dos fatores [multiplicados] três a três é $\frac{-1}{1.2 .3 . y}$; a soma de todos os fatores [multiplicados] quatro a quatro é $=0$; a soma dos fatores [multiplicados] cinco a cinco $=\frac{+1}{1.2 \cdot 3 \cdot 4 \cdot 5 \cdot y}$; a soma dos fatores [multiplicados] seis a seis $=0$. $\mathrm{E}$ assim por diante.

§.8. Mas se é tomada uma série qualquer $\mathrm{a}+\mathrm{b}+\mathrm{c}+\mathrm{d}+\mathrm{e}+\mathrm{f}+$ etc. cuja soma seja $\alpha$, a soma dos fatores [multiplicados] dois a dois $=\beta$; a soma dos fatores [multiplicados] três a três $=\gamma$; a soma dos fatores [multiplicados] quatro a quatro $=\delta$ etc., a soma dos quadrados

\footnotetext{
${ }^{3}$ No original, Euler se engana e escreve “ex quaternis”, "tomados quatro a quatro". 
de cada termo, isto é, $\mathrm{a}^{2}+\mathrm{b}^{2}+\mathrm{c}^{2}+\mathrm{d}^{2}+$ etc. será $=\alpha^{2}-2 \beta$, e a soma dos cubos $\mathrm{a}^{3}+\mathrm{b}^{3}+\mathrm{c}^{3}$ $+\mathrm{d}^{3}+$ etc. $=\alpha^{3}-3 \alpha \beta+3 \gamma$; a soma dos biquadrados $=\alpha^{4}-4 \alpha^{2} \beta+4 \alpha \gamma+2 \beta^{2}-4 \delta$. Mas para que fique mais claro como estas fórmulas progridem, façamos a soma dos próprios termos a, b, c, d, etc. ser $=$ P, a soma dos quadrados $=\mathrm{Q}$, a soma dos cubos $=\mathrm{R}$, a soma dos biquadrados $=\mathrm{S}$, a soma das quintas potências $=\mathrm{T}$, a soma das sextas $=\mathrm{V}$, etc. Feito isso, será $\mathrm{P}=\alpha ; \mathrm{Q}=\mathrm{P} \alpha-2 \beta ; \mathrm{R}=\mathrm{Q} \alpha-\mathrm{P} \beta+3 \gamma ; \mathrm{S}=\mathrm{R} \alpha-\mathrm{Q} \beta+\mathrm{P} \gamma+4 \delta ; \mathrm{T}=\mathrm{S} \alpha-\mathrm{R} \beta+\mathrm{Q} \gamma-\mathrm{P} \delta$ $+5 \varepsilon$; etc.

§.9. Como, portanto, em nosso caso, a soma de todos os termos da série $\frac{1}{A}, \frac{1}{p-A}$, $\frac{1}{-p-A}, \frac{1}{2 \mathrm{p}+\mathrm{A}}, \frac{1}{-2 \mathrm{p}+\mathrm{A}}, \frac{1}{3 p-A}, \frac{1}{-3 p-A}$, etc. é $\alpha=\frac{1}{y}$; a soma dos fatores [multiplicados] dois a dois é $\beta=0$ e os seguintes $\gamma=\frac{-1}{1.2 .3 \cdot y}, \delta=0, \varepsilon=\frac{+1}{1.2 .3 .4 .5 . y}, \zeta=$ 0 , etc., a soma dos próprios termos será $\mathrm{P}=\frac{1}{y}$; a soma dos quadrados dos termos $\mathrm{Q}=\frac{P}{y}=$ $\frac{1}{y^{2}}$; a soma dos cubos dos termos $\mathrm{R}=\frac{Q}{y}-\frac{1}{1.2 y}$; a soma dos biquadrados $\mathrm{S}=\frac{R}{y}-\frac{P}{1.2 .3 y}$ . E ainda $\mathrm{T}=\frac{S}{y}-\frac{Q}{1.2 .3 y}+\frac{1}{1.2 .3 .4 y} ; \mathrm{V}=\frac{T}{y}-\frac{R}{1.2 .3 y}+\frac{P}{1.2 .3 .4 .5 y} ; \mathrm{W}=\frac{V}{y}-$ $\frac{S}{1.2 .3 y}+\frac{Q}{1.2 .3 .4 .5 y}-\frac{1}{1.2 .3 .4 .5 .6 y}$. A partir desta lei, as somas das potências mais altas restantes são determinadas facilmente.

§.10. Façamos agora o seno $\mathrm{PM}=y$ igual ao raio, tal que $y=1$, e será o arco mínimo A, cujo seno é 1 , a quarta parte da periferia, $=\frac{1}{2} p$, ou, denotando por $q$ a quarta parte da periferia, será $\mathrm{A}=q$ e $p=2 q$. E daí que a série anterior se transforma nesta $\frac{1}{q}, \frac{1}{q},-\frac{1}{3 q}$, $\frac{1}{3 q}, \frac{+1}{5 q}, \frac{+1}{5 q},-\frac{1}{7 q},-\frac{1}{7 q}, \frac{+1}{9 q}, \frac{+1}{9 q}$, etc. com termos iguais dois a dois. Daí que a soma destes termos, que é $\frac{2}{q}\left(1-\frac{1}{3}+\frac{1}{5}-\frac{1}{7}+\frac{1}{9}-\frac{1}{11}+e t c.\right)$ é igual a $\mathrm{P}=1$. Daqui vem, portanto, que $1-\frac{1}{3}+\frac{1}{5}-\frac{1}{7}+\frac{1}{9}-\frac{1}{11}+e t c$. é $=\frac{q}{2}=\frac{p}{4}$. Então o quádruplo desta série é igual à semiperiferia do círculo cujo raio é 1 , ou a toda a periferia do círculo, cujo diâmetro é 1 . Esta é a série já tratada por Leibniz, com a qual ele encontrou a quadratura do círculo. Com isso, fica claro o fundamento sólido deste método, se a alguém ele talvez não pareça certo; assim também não se pode em absoluto duvidar das outras coisas que se derivam desse método. 
$\S .11$. Somemos agora o quadrado dos termos encontrados para o caso $y=1$, e vem esta série $\frac{+1}{q^{2}}+\frac{1}{q^{2}}+\frac{1}{9 q^{2}}+\frac{1}{9 q^{2}}+\frac{1}{25 q^{2}}+\frac{1}{25 q^{2}}+$ etc . cuja soma é $\frac{1}{q^{2}}\left(\frac{1}{1}+\frac{1}{9}+\frac{1}{25}+\frac{1}{49}+e t c\right.$.) que, portanto, deve ser igual a $\mathrm{Q}=\mathrm{P}=1$. Daí segue que a soma da série $1+\frac{1}{9}+\frac{1}{25}+\frac{1}{49}+e t c$. é $=\frac{q}{2}=\frac{p^{2}}{4}$ denotando como $p$ toda a periferia do círculo cujo diâmetro é 1 . Mas a soma desta série $1+\frac{1}{9}+\frac{1}{25}+e t c$. depende da soma da série $1+\frac{1}{4}+\frac{1}{9}+\frac{1}{16}+\frac{1}{25}+e t c$. porque esta diminuída de uma quarta parte sua dá aquela. E a soma desta série é igual à soma daquela com seu terço. Por isso, será $1+\frac{1}{4}+\frac{1}{9}+\frac{1}{16}+\frac{1}{25}+\frac{1}{36}+e t c .=\frac{p^{2}}{6}$ e, portanto, a soma desta série multiplicada por 6 é igual ao quadrado da periferia do círculo cujo diâmetro é 1; esta é a proposição que mencionei o início.

§.12. Como no caso em que $y=1$ temos que $\mathrm{P}=1$ e $\mathrm{Q}=1$, os [valores] das letras restantes $\mathrm{R}, \mathrm{S}, \mathrm{T}, \mathrm{V}$ etc. serão como a seguir: $\mathrm{R}=\frac{1}{2} ; \mathrm{S}=\frac{1}{3} ; \mathrm{T}=\frac{5}{24} ; \mathrm{V}=\frac{2}{15} ; \mathrm{W}=\frac{61}{720} ; \mathrm{X}=\frac{17}{325}$; etc. Como a soma dos cubos é igual a $\mathrm{R}=\frac{1}{2}$, será $\frac{2}{q^{3}}\left(1-\frac{1}{3^{3}}+\frac{1}{5^{3}}-\frac{1}{7^{3}}+\frac{1}{9^{3}}-e t c.\right)=\frac{1}{2}$. Por isso, será $1-\frac{1}{3^{3}}+\frac{1}{5^{3}}-\frac{1}{7^{3}}+\frac{1}{9^{3}}-e t c .=\frac{q^{3}}{4}=\frac{p^{3}}{32}$. Assim, a soma dessa série multiplicada por 32 dá o cubo da periferia do círculo cujo diâmetro é 1 . De maneira semelhante, a soma dos biquadrados, que é $\frac{2}{p^{4}}\left(1+\frac{1}{3^{4}}+\frac{1}{5^{4}}+\frac{1}{7^{4}}+\frac{1}{9^{4}}+e t c.\right)$ deve ser igual a $\frac{1}{2}$, e por isso será $1+\frac{1}{3^{4}}+\frac{1}{5^{4}}+\frac{1}{7^{4}}+\frac{1}{9^{4}}+e t c .=\frac{q^{4}}{6}=\frac{p^{4}}{96}$. E esta série multiplicada por $\frac{16}{15}$ é igual a $1+\frac{1}{2^{4}}+\frac{1}{3^{4}}+\frac{1}{5^{4}}+\frac{1}{6^{4}}+e t c$., e por isso essa série é igual a $\frac{p^{4}}{90}$; ou soma da série $1+\frac{1}{2^{4}}+\frac{1}{3^{4}}+\frac{1}{4^{4}}+e t c$. multiplicada por 90 dá o biquadrado da periferia do círculo cujo diâmetro é 1 .

§.13. De maneira semelhante, serão encontradas as somas das potências superiores, mostradas como a seguir: $1-\frac{1}{3^{5}}+\frac{1}{5^{5}}-\frac{1}{7^{5}}+\frac{1}{9^{5}}-e t c .=\frac{5 q^{5}}{48}=\frac{5 p^{5}}{1536}$; e $1+\frac{1}{3^{6}}+\frac{1}{5^{6}}+\frac{1}{7^{6}}+\frac{1}{9^{6}}+e t c \cdot=\frac{q^{6}}{15}=\frac{p^{6}}{960}$. Encontrada a soma desta série, será conhecida ao mesmo tempo a soma da série $1+\frac{1}{2^{6}}+\frac{1}{3^{6}}+\frac{1}{4^{6}}+\frac{1}{5^{6}}+e t c$. que será $\frac{p^{6}}{945}$. Em seguida, para a 
sétima potência, será $1-\frac{1}{3^{7}}+\frac{1}{5^{7}}-\frac{1}{7^{7}}+\frac{1}{9^{7}}-e t c .=\frac{61 q^{7}}{1440}=\frac{61 p^{7}}{184320}$ e para a oitava, $1+\frac{1}{3^{8}}+\frac{1}{5^{8}}+\frac{1}{7^{8}}+\frac{1}{9^{8}}+e t c=\frac{17 q^{8}}{630}=\frac{17 p^{8}}{161280} ;$ de onde se deduz que $1+\frac{1}{2^{8}}+\frac{1}{3^{8}}+\frac{1}{4^{8}}+\frac{1}{5^{8}}+\frac{1}{6^{8}}+e t c .=\frac{p^{8}}{9450}$. Devemos observar que os sinais dos termos das séries de potências de expoentes ímpares se alternam e, para as potências pares, são iguais; e esta é a causa por que a soma da série geral $1+\frac{1}{2^{n}}+\frac{1}{3^{n}}+\frac{1}{4^{n}}+e t c$. só pode ser dada naqueles casos em que $n$ é um número par. Além disso, devemos notar também que se pode ser encontrado o termo geral da série $1,1, \frac{1}{2}, \frac{1}{3}, \frac{5}{24}, \frac{2}{15}, \frac{61}{720}, \frac{17}{325}$ etc., valores que encontramos para as letras $\mathrm{P}, \mathrm{Q}, \mathrm{R}, \mathrm{S}$ etc., então a quadratura do círculo será encontrada.

§.14. Para isso, façamos o seno PM ser igual ao raio e vejamos então quais séries surgem quando são atribuídos outros valores a $y$. Seja, portanto, $y=\frac{1}{\sqrt{2}}$, a cujo seno o arco mínimo correspondente é $\frac{1}{4} \mathrm{p}$. Posto então $\mathrm{A}=\frac{1}{4} \mathrm{p}$, a série de termos simples ou de primeira potência será $\frac{4}{p}+\frac{4}{3 p}-\frac{4}{5 p}-\frac{4}{7 p}+\frac{4}{9 p}+\frac{4}{11 p}-e t c$. da qual s soma P é igual a $\frac{1}{y}=\sqrt{2}$. Portanto, se terá $\frac{p}{2 \sqrt{2}}=1+\frac{1}{3}-\frac{1}{5}-\frac{1}{7}-\frac{1}{9}-\frac{1}{11}-\frac{1}{13}-\frac{1}{15}-$ etc ., série que difere da de Leibniz só na disposição dos sinais, e que já foi há muito tratada por Newton. E a soma dos quadrados daqueles termos, a saber, $\frac{16}{p^{2}}\left(1+\frac{1}{9}+\frac{1}{25}+\frac{1}{49}+e t c\right.$. é igual a $\mathrm{Q}=2$. Portanto, será $1+\frac{1}{9}+\frac{1}{25}+\frac{1}{49}+e t c .=\frac{p^{2}}{8}$, como foi encontrado antes.

§.15. Se é feito $y=\frac{\sqrt{3}}{2}$, o arco mínimo correspondente a esse seno será $60^{\circ}$ e, por isso, $A=\frac{1}{3} p$. Neste caso, vem a seguinte série de termos $\frac{3}{p}+\frac{3}{2 p}-\frac{3}{4 p}-\frac{3}{5 p}+\frac{3}{7 p}+\frac{3}{8 p}-e t c$., cuja soma dos termos é igual a $\frac{1}{y}=\frac{2}{\sqrt{3}}$. Então será $\frac{2 p}{3 \sqrt{3}}=1+\frac{1}{2}-\frac{1}{4}-\frac{1}{5}+\frac{1}{7}-\frac{1}{8}-\frac{1}{10}-\frac{1}{11}+$ etc. A soma dos quadrados desses termos é $=\frac{1}{y^{2}}=\frac{4}{3}$; de onde vem que $\frac{4 p^{2}}{27}=1+\frac{1}{4}+\frac{1}{16}+\frac{1}{25}+\frac{1}{49}+\frac{1}{64}+e t c$., série da qual falta cada terceiro termo. Mas esta série depende também desta $1+\frac{1}{4}+\frac{1}{9}+\frac{1}{16}+e t c$. cuja 
soma havia sido encontrada $=\frac{p^{2}}{6}$; pois se desta série se diminui uma nona parte sua vem a série anterior, cuja soma, por isso, deve ser $=\frac{p^{2}}{6}\left(1-\frac{1}{9}\right)=\frac{4 p p}{27}$. De modo semelhante, se são tomados outros senos, outras séries surgirão, tanto de termos simples quanto de termos quadrados e de potências mais altas cujas somas envolvem a quadratura do círculo.

§.16. Mas se é posto $y=0$, as séries não poderão mais ter somas por causa do $y$ no denominador, ou a equação inicial dividida por $y$. Mas séries poderão ser deduzidas daí de outro modo se $n$ é um número par na série $1+\frac{1}{2^{n}}+\frac{1}{3^{n}}+\frac{1}{4^{n}}+$ etc .; assim, encontradas as somas destas séries, deduzirei depois o caso em que $y=0$. Então, posto $y=0$, a mesma equação fundamental se torna $0=s-\frac{s^{3}}{1.2 .3 .}+\frac{s^{5}}{1.2 .3 .4 .5}-\frac{s^{7}}{1.2 .3 .4 .5 .6 .7}+$ etc ., equação cujas raízes dão todos os arcos dos quais o seno é $=0$. Mas há uma só raiz mínima $s=0$ e, por isso, a equação, dividida por $s$, mostrará todos os arcos restantes cujo seno é = 0 ; esses arcos serão as raízes desta equação $0=1-\frac{s^{2}}{1.2 .3 .}+\frac{s^{4}}{1.2 .3 .4 .5}-\frac{s^{6}}{1.2 \cdot 3 \cdot 4 \cdot 5.6 .7}+e t c$. E os arcos dos quais o seno é $=0$ são p, $-\mathrm{p},+2 \mathrm{p},-2 \mathrm{p},+3 \mathrm{p},-3 \mathrm{p}$ etc., em que um elemento a cada dois é o negativo do outro, e isso porque a mesma equação, por causa das dimensões de $s$, só mostra os pares. Por isso, os divisores daquela equação serão $1-\frac{s}{p}, 1+\frac{s}{p}, 1-\frac{s}{2 p}, 1+\frac{s}{2 p}$, etc., e com esses divisores conjugados dois a d dois teremos $1-\frac{s^{2}}{1.2 .3}+\frac{s^{4}}{1.2 .3 .4 .5}-\frac{s^{6}}{1.2 .3 .4 .5 .6 .7}+e t c .=\left(1-\frac{s^{2}}{p^{2}}\right)\left(1-\frac{s^{2}}{4 p^{2}}\right)\left(1-\frac{s^{2}}{9 p^{2}}\right)\left(1-\frac{s^{2}}{16 p^{2}}\right)$ etc .

§.17. Já está manifesto, pela natureza das equações, que o coeficiente $\frac{1}{1.2 .3}$ de ss será igual a $\frac{1}{p^{2}}+\frac{1}{4 p^{2}}+\frac{1}{9 p^{2}}+\frac{1}{16 p^{2}}+e t c$. E a soma dos fatores desta série [multiplicados] dois a dois será $=\frac{1}{1.2 .3 .4 .5}$; e a soma dos fatores [multiplicados] três a três etc. Por essa razão, será, como no $\S .8, \alpha=\frac{1}{1.2 .3} ; \beta=\frac{1}{1.2 .3 .4 .5} ; \gamma=\frac{1}{1.2 .3 .4 .5 .6 .7}$, etc., e também que, posta a soma dos termos $\frac{1}{p^{2}}+\frac{1}{4 p^{2}}+\frac{1}{9 p^{2}}+\frac{1}{16 p^{2}}+e t c .=\mathrm{P}$, e a soma dos quadrados daqueles termos $=\mathrm{Q}$; a soma dos cubos $=\mathrm{R}$; a soma dos biquadrados $=\mathrm{S}$; etc., será, pelo $\S .8, \mathrm{P}=\alpha=\frac{1}{1.2 .3}=\frac{1}{6} ; \mathrm{Q}=\mathrm{P} \alpha-2 \beta=\frac{1}{90} ; \mathrm{R}=\mathrm{Q} \alpha-\mathrm{P} \beta+3 \gamma=\frac{1}{945} ; \mathrm{S}=\mathrm{R} \alpha-\mathrm{Q} \beta+\mathrm{P} \gamma-$ 
$4 \delta=\frac{1}{9450} ; \mathrm{T}=\mathrm{S} \alpha-\mathrm{R} \beta+\mathrm{Q} \gamma-\mathrm{P} \delta+5 \varepsilon=\frac{1}{93555} ; \mathrm{V}=\mathrm{T} \alpha-\mathrm{S} \beta+\mathrm{R} \gamma-\mathrm{Q} \delta+\mathrm{P} \varepsilon-6 \zeta=$ $\frac{691}{6825.93555}$ etc.

§.18. Daí, portanto, as seguintes somas são derivadas:

$$
\begin{aligned}
& 1+\frac{1}{2^{2}}+\frac{1}{3^{2}}+\frac{1}{4^{2}}+\frac{1}{5^{2}}+e t c=\frac{p^{2}}{6}=\mathrm{P} \\
& 1+\frac{1}{2^{4}}+\frac{1}{3^{4}}+\frac{1}{4^{4}}+\frac{1}{5^{4}}+\text { etc }=\frac{p^{4}}{90}=\mathrm{Q} \\
& 1+\frac{1}{2^{6}}+\frac{1}{3^{6}}+\frac{1}{4^{6}}+\frac{1}{5^{6}}+\text { etc } .=\frac{p^{6}}{945}=\mathrm{R} \\
& 1+\frac{1}{2^{8}}+\frac{1}{3^{8}}+\frac{1}{4^{8}}+\frac{1}{5^{8}}+\text { etc }=\frac{p^{8}}{9450}=\mathrm{S} \\
& 1+\frac{1}{2^{10}}+\frac{1}{3^{10}}+\frac{1}{4^{10}}+\frac{1}{5^{10}}+\text { etc }=\frac{p^{10}}{93555}=\mathrm{T} \\
& 1+\frac{1}{2^{12}}+\frac{1}{3^{12}}+\frac{1}{4^{12}}+\frac{1}{5^{12}}+\text { etc }=\frac{691 p^{12}}{6825.93555}=\mathrm{V} .
\end{aligned}
$$

Com a lei dada, porém, só com muito trabalho essas séries podem ser estendidas para potências mais altas. Mas, dividindo cada série pela precedente, surgem as seguintes equações: $p^{2}=6 \mathrm{P}=\frac{15 Q}{P}=\frac{21 R}{2 Q}=\frac{10 \mathrm{~S}}{R}=\frac{99 \mathrm{~T}}{10 \mathrm{~S}}=\frac{6825 \mathrm{~V}}{691 \mathrm{~T}}$ etc., e cada uma dessas expressões é igual ao quadrado da periferia do círculo cujo diâmetro é 1.

§.19. Ainda que as somas dessas séries possam ser exibidas facilmente, elas não são de muita utilidade para exprimir aproximadamente a periferia do círculo por causa da raiz quadrada que deverá ser extraída. Das séries anteriores, vamos derivar expressões que sejam iguais à própria periferia $p$. E elas são como se segue:

$$
\begin{aligned}
& p=4\left(1-\frac{1}{3}+\frac{1}{5}-\frac{1}{7}+\frac{1}{9}-\frac{1}{11}+e t c .\right) \\
& p=2\left(\frac{1+\frac{1}{3^{2}}+\frac{1}{5^{2}}+\frac{1}{7^{2}}+\frac{1}{9^{2}}+\frac{1}{11^{2}}+e t c .}{1-\frac{1}{3}+\frac{1}{5}-\frac{1}{7}+\frac{1}{9}-\frac{1}{11}+e t c .}\right)
\end{aligned}
$$

RBHM, Vol. 21, n 42, pp. 206-228, 2021 
Frederico J. A. Lopes

$$
p=4\left(\begin{array}{c}
\frac{1-\frac{1}{3^{3}}+\frac{1}{5^{3}}-\frac{1}{7^{3}}+\frac{1}{9^{3}}-\frac{1}{11^{2}}+e t c .}{1+\frac{1}{3^{2}}+\frac{1}{5^{2}}+\frac{1}{7^{2}}+\frac{1}{9^{2}}+\frac{1}{11^{2}}+e t c .} \\
p=\frac{16}{5}\left(\begin{array}{c}
\frac{1}{3^{4}}+\frac{1}{5^{4}}+\frac{1}{7^{4}}+\frac{1}{9^{4}}+\frac{1}{11^{4}}+e t c . \\
\frac{1-\frac{1}{3^{3}}+\frac{1}{5^{3}}-\frac{1}{7^{3}}+\frac{1}{9^{3}}-\frac{1}{11^{3}}+e t c .}{1+\frac{1}{3^{5}}+\frac{1}{5^{5}}-\frac{1}{7^{5}}+\frac{1}{5^{5}}-\frac{1}{7^{4}}+\frac{1}{9^{4}}+\frac{1}{11^{5}}+e t c .}
\end{array}\right) \\
\frac{192}{1+\frac{1}{3^{6}}+\frac{1}{5^{6}}+\frac{1}{7^{6}}+\frac{1}{9^{6}}+\frac{1}{11^{6}}+e t c .} \\
\frac{1-\frac{1}{3^{5}}+\frac{1}{5^{5}}-\frac{1}{7^{5}}+\frac{1}{9^{5}}-\frac{1}{11^{5}}+e t c .}{1-\frac{1}{3^{7}}+\frac{1}{5^{7}}-\frac{1}{7^{7}}+\frac{1}{9^{7}}-\frac{1}{11^{7}}+e t c .}
\end{array}\right)
$$




\title{
3. Texto original
}

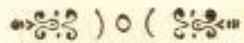 \\ DE SVMMIS SERIERVM RECIPROCARVM.}

123

\author{
AVCTORE
}

Leoub. Eulero.

5. $x$.

$\mathrm{T}$

Antopere iam pertractatac et inueftigatae funt fe- $\mathbf{T}$ abula vis

ries reciprocae poteftatum numerorum nattira-

lium, vt vix probabile videatur de iis noui quicquam inueniri poffe. Quicunque enim de fummis ferierum medicati funt, ii fere omnes quoque in fummas huitsmodi ferierum inquifiuerunt, neque tamen vlla methodo eas idoneo modo exprimere potueruut. Ego ctiam iam faepius, cum varias fummandi methodos tradidffem, has feries diligenter fum perfecutus, neque tamen quicquam aliud fum affecutus, nifi vt earum fummam vel proxime veram definiuerim vel ad quadraturas curuarum maxime tranfcendentium reduxcrim; quorum illud in differtatione proxime praclect.t, hoc vero in priecedentibus praeftiti. Loquor hic autem de fcriebus fractionum, quarum numeratores funt 1 , denominatores vero vel quadrata, vel cubi, vel aliae dignitates numerorum naturalium; cuius modi funt $\mathrm{I}+\mathrm{i}-\mathrm{+}-\mathrm{i}$ $+\frac{1}{2}+\frac{1}{23}+$ etc., item $\mathrm{I}+\frac{1}{3}+\frac{1}{4}+\frac{1}{4}+$ etc. atque fimiles fuperiorum poteftatum, quarum termini gencrules continentur in hac forma $\frac{1}{x^{n}}$.

Q 2

\$. 2 .

RBHM, Vol. 21, n 42, pp. 206-228, 2021 


\section{$1=4 \quad$ DE SVMMIS}

6. 2. Deductus fum autem nuper omnino inopinato ad elegantem fummae huius feriei $\mathrm{I}+\frac{1}{3}+\frac{1}{5}+\frac{1}{\mathrm{~s}}$ +-etc. expreflionem, quae a circuli quadratura pendet, ita, ve fi huius feriei vera fumma haberetur, inde fimul circuli quadratura fequeretur. Inueni enim fummac huius feriei fextuplum aequale effe quadrato peripheriac circuli, cuins diameter eft $\mathbf{x}$; feu pofita iftius feriei fum$\mathrm{ma}=s$, tenebit $\sqrt{ } 6 s$ ad 1 rationem peripheriae ad diametrum. Huius autem feriei fummam nuper oftendi proxime effe I, 6449340668482264364 , ex cuits numeri fextuplo, fi extrahatur radix quadrata, reipfa prodit numerus $3, x_{41} 592653589793238$ exprimens circuli peripheriam, cuius diameter eft 1 . Iisdam porro veftigiis, quibus hane fummam fum confecutus, incedens huius feriei $\mathrm{x}+\frac{1}{16}+\frac{1}{x^{2}}+\frac{1}{25}+\frac{5}{x^{5}}+$ etc. fummam quoque a quadratura circuli pendere deprehendi. Summa nempe eius per 90 multiplicata dat biquadratum peripheriae circuli, cuius diameter eft I. Atque fimili matione etiam fequentium ferierum, in quibus exponentes dignitatum funt numeri pares, fummas determinare potui.

§. 3. Quo igitur, quemadmodum haec fam adeptus, commodifime oftendam, totam rem, quo ipfe vfus fum, ordine exponam. In circulo AMBNA centro C sigua I. radio $A C$ vel $B C=x$ defcripto contemplatus fum arcum quemcunque AM, cuius finus eft $M P$, cofinus vero $\mathrm{CP}$. Pofito nunc arcu $\mathrm{AM}=s$, fim $\mathrm{PM}=y$, ct cofinu $\mathrm{CP}=x$, per methodum iam fatis cognitam tam finus $y$ quam cofinus $x$ ex dato arcu $s$ per feries pof- 
funt definiri, eft enim, vti paffim videre licet $y=s-$

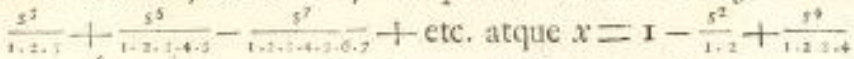
- $\frac{s^{5}}{1}+$ etc. Ex harum feilicet aequationum confideratione ad fummas fupra memoratarum ferierum reciprocarum perueni ; quarum aequationum quidem vtraque ad eundem fere fcopum dirigitur, et hane ob rem fifficiet altersm tantum co, quen fim expofiturus, modo tructufe.

6. 4. Aequatio ergo prior $y=s-\frac{s^{3}}{1+2 \cdot 3}+\frac{s^{s}}{1 \cdot 2 \cdot+\cdot+-5}$ $\frac{5}{5.6}-$ - etc. exprimit relationem inter arcum et finum. Quare ex ea tam ex dato arcu eius funs, quam ex dato finu eius arcus determinari poterit. Confidero autem finum $y$ tanquam datum, et inteftigo, quemadmodum arcum s ex $y$ crui oporteat. Hic vero ante omnia animaduertendum eft, eidem finui $y$ innumerabiles arcus refpondere, quos ergo innumerabiles arcus aequatio propofita praebere debebit. $\mathrm{Si}$ quidem in ifts aequatione s tanquam incognita fpectetur, ea infinitas habet dimenfiones, ideoque mirum non eft, fi ifta aequatio innumeros contineat factores fimplices, quorum quisque niliilo aequalis pofitas, idoneum pros valorem dure debet.

§. 5. Quemadmodum autem, fi omnes fictores huius aequationis cognitı effent, omnes quogue radices illits fea valores ipfus s innotefcerent, ita vicifim fi omnes valores iplius s affigaari poterunt, tum quoque ipfi fictores omnes habebuntur. Quo autem eo melius tam de radicibus quam de factoribus iudicare queam, trans-

$$
\text { Q } 3
$$

muto 
muto aequationem propofitam in hanc formam: $0=a-$ $\frac{s}{y}+\frac{s^{5}}{1 \cdot 2 \cdot 5 \cdot y}-\frac{s^{5}}{1 \cdot 2 \cdot 2 \cdot+\cdot 5 \cdot y}+$ etc. Si nunc omnes radices huus acquationis feu omnes arcus, quorum idem eft finus $y$, fuerint $\mathrm{A}, \mathrm{B}, \mathrm{C}, \mathrm{D}, \mathrm{E}$ etc. tum tactores quoque erunt omnes iftac quantitates, $I-\frac{5}{A}, I-\frac{5}{B}, I-\frac{s}{C}, I-\frac{8}{D}$ etc. Quamobrem erit $1-\frac{5}{y}+\frac{s^{2}}{1 \cdot 2 \cdot 5 y}-\frac{s^{2}}{1+2 \cdot 5 \cdot+\cdot 5 \cdot y}+$-ctc. $=\left(\mathrm{I}-\frac{\mathrm{s}}{\mathrm{A}}\right)\left(\mathrm{I}-\frac{\mathrm{s}}{\mathrm{B}}\right)\left(\mathrm{I}-\frac{\mathrm{s}}{\mathrm{C}}\right)\left(\mathrm{x}-\frac{3}{\mathrm{D}}\right) \mathrm{etc}$.

§. 6. Ex natura autem et refolutione aequationum conftat, effe coëfficientem termini, in quo inceft $s$, feu $\frac{1}{y}$ aequalem fumanc omnium coëfficientium ipfus $s$ in factoribus feu $\frac{1}{y}=\frac{1}{A}+\frac{1}{\mathrm{~B}}+\frac{1}{\mathrm{C}}+\frac{\mathrm{b}}{\mathrm{D}}+\mathrm{etc}$. Deinde eft coëfficiens ipfuus $s^{2}$, qui eft $=0$, ob hunc terminum in aequatione deficientem, aequalis aggregato factorum ex binis terminis feriei, $\frac{1}{A}, \frac{1}{B}, \frac{1}{C}, \frac{b}{b}$ etc. Porro erit $\frac{1}{2.3-y}$ aequale aggregato factorum ex quaternis terminis eiusdem feriei $\frac{1}{A}, \frac{b}{b}, \frac{1}{C}, \frac{x}{D}$ etc. Similique modo erit $0=$ aggregato fictorum ex quaternis tcrminis ciusdem feriei, et $+\frac{1}{1,7+5.5}=$ aggregato factorum ex quinis terminis iftius feriei, et ita porro.

\$. 7. Pofito autem minimo arcu $\mathrm{A} M=\mathrm{A}$, cuius finus eft $\mathrm{P} M=y$, et femiperipheria circuli $=p$, erunt $\mathbf{A}, p-\mathbf{A}, 2 p+\mathbf{A}, 3 p-\mathbf{A}, 4 p+\mathbf{A}, 5 p-\mathbf{A}, 6 p+\mathbf{A}$ etc. item $-p-\mathrm{A},-2 p+\mathrm{A},-3 p-\mathrm{A},-4 p-\mathrm{A}$, $-5 p-\mathrm{A}$, etc. omnes arcus, quorum finus eft idem $y$. Qram igitur ante affumfimus feriem $\frac{i}{\hat{A}}, \dot{b}, \dot{c}, \dot{\mathrm{D}}, \mathrm{ctc}, \mathrm{ca}$

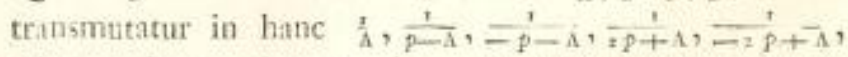
$\frac{1}{p-\Lambda}, \frac{1}{p-s p-\Lambda}, \frac{1}{+p+\alpha}, \frac{1}{-+p+\Lambda}$ ctc. Hurum ergo omnium

termi- 


\section{SERIERIM RECIPROCARVM. $\mathrm{x}=\mathrm{\psi}$}

ecrminorum fumma cft $=\frac{t}{y}$; fumma autem fictorum ex binis terminis hutus feriei eft aequalis o; fumma factorum ex ternis $=\frac{-1}{1,2+y}$, fumma factorum ex quaternis $=0$; fumma fictorum ex quinis $=\frac{+t}{1,2 .}+\frac{1}{4}$; fumma factorum ex écnis $=0$. Atque ita porro.

6. 8. Si autem habeatur feries quaecunque $a+b$ $+c+d+e+f+c t c$. cuius fumma fit $a$, fumma facturum ex binis terminis $=b$; fumma fictorum ex teriais $=\gamma$; fumma fictorum ex quaternis $=\delta$, etc. erit fumma quadratorum fingulorum terminorum, hoc eft $a^{2}+b^{2}+c^{2}+d^{2}+$ etc. $=a^{2}-2 b$; fumma vero cuborum $a^{3}+b^{3}+c^{3}+d^{2}$ etc $=a^{3}-3 a b^{b}+3 \gamma$; fumma biquadratorum $=a^{2}-4 \alpha^{2} b^{2}+4 a \gamma+2 a^{2}-4 \delta$. Quo autem clatius appareat, quomodo hae formulae progrediantur, ponamus ipforum terminorum $a, b, c, d$, etc. fummam effe $=\mathrm{P}_{\text {, fimmam quadratorum }}=\mathrm{Q}$, fummam cuborum $=R$, fimmam biquadratotum $=S$, fum m:1m poteftum quint:rum $=\mathrm{T}$, fummam fextarum $=\mathrm{V}$ etc. His pofitis erit $\mathrm{P}=\alpha ; \mathrm{Q}=\mathrm{P} \alpha-=\mathrm{b} ; \mathrm{R}=\mathrm{Q} \alpha-$ $\mathrm{Pb}+3 \gamma ; \mathrm{S}=\mathrm{R} \alpha-\mathrm{Q} b+\mathrm{P} \gamma+4 \delta ; \mathrm{T}=\mathrm{S} \alpha-\mathrm{R} b$ $+\mathrm{Q} \gamma-\mathrm{P} d+5 \varepsilon ;$ etc.

6. 9. Cum igitur in noftro cafu ferie $\frac{1}{A}, \frac{\gamma}{p-1}, \frac{1}{-p-A}$ $\frac{t}{p+1}, \frac{1}{-p+1}, \frac{1}{p-A},=\frac{1}{p-A}$ etc. fumma omnium terminorum fea $\alpha$ fit $=\frac{5}{y}$; fumma factorum ex binis fea

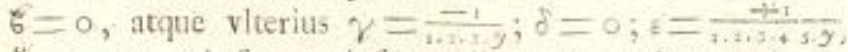
$\xi=0$; etc erit fumma ipforum illorum terminorum $\mathrm{P}=3$; famma quadratorum illorum terminorum $Q=\frac{p}{y}=\frac{1}{y^{2}}$; 
fumma cuborum illorum terminorum $R: \frac{0}{y}-\frac{1}{t: 2-y}$; fum$\mathrm{ma}$ biquadratorum $\mathrm{S}=\frac{\mathrm{R}}{y}-\frac{\mathrm{P}}{\mathrm{y}}$. Atque porro $\mathrm{T}=\frac{\mathrm{S}}{\mathrm{y}}$

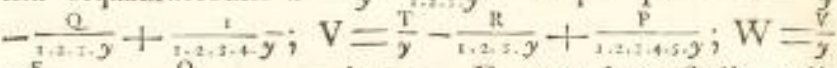
$\frac{-5}{1.2+y}+\frac{2}{1.5+4.5 y}-\frac{1}{1.5+5.5 .6 y}$. Ex qua lege facile reliquarum altiorum poteftatum fummae determinantur.

6. 10. Ponamus nunc finum $\mathrm{PM}=y$ aequalem radio, vt fit $y=1$, erit minimus arcus A cuius finus eft I quarta peripheriae pars, $=\frac{1}{2} p$, feu denotante $q$ quartam peripheriae partem erit $\mathrm{A}=q$ ct $p=2 q$. Superior ergo ferics abibit in iftam $\frac{x}{q}, \frac{x}{q}, \frac{-1}{1 q},-\frac{1}{1 q}, \frac{+1}{5 q},+\frac{1}{s q},-\frac{x}{q q}$, $-\frac{1}{g q}, 十_{9 q}^{1}, 十_{9 q}^{b}$, etc. binis terminis exiftentibas aequalibus. Horum ergo terminorum fumma, quae eft $\frac{t}{q}$ $\left(\mathrm{x}-\frac{1}{3}+\frac{1}{2}-\frac{1}{7}+\frac{1}{6}-\frac{1}{x}+\right.$ etc. $)$ aequalis eft ipfi $\mathrm{P}=\mathrm{x}$. Hinc igitur oritur $1-\frac{1}{3}+\frac{1}{3}-\frac{1}{3}+\frac{1}{3}-\frac{1}{12}+$ etc. $=q=?$ ? Huius ergo feriei quadruplum aequatur femiperipheriae circuli, cuius radius eft $\mathrm{I}$, feu toti peripheriae circuli, cuius diameter eft $\mathrm{i}$. Atque haec eft ipfa feries a Leibnitio iam pridem prolata, qua circuli quadraturam definiuit. Ex quo magnum huius methodi, fi cui forte ea non fatis certa videatur, firmamentum clucet; ita vt de reliquis, quae ex hac methodo deriuabantur, omnino non liceat dubitari.

6. II. Sumamus nunc intentorum terminorum pro catu quo $y=\mathrm{r}$, quadrata, prodibitgue haec feries $+\frac{\mathrm{x}}{q^{2}}$ $+\frac{1}{9^{2}}+\frac{1}{59^{2}}+\frac{1}{99^{3}}+\frac{1}{25 q^{y}}+\frac{1}{25 q^{2}}+$ etc. cuins fumma eft $\overrightarrow{q^{2}}\left(\frac{1}{1}+\frac{1}{y}+\frac{1}{2}+\frac{1}{+y}+\right.$ ctc. $)$, qua ergo aequalis cfle dcbet ipfi $Q=P=x$. Ex quo fequitur huius ferici $x+\frac{3}{9}$ 
$+\frac{1}{15}+\frac{1}{99}+$ etc. fummam effe $=q_{2}^{2}=p_{2}^{2} ;$ denotante $p$ totam circuli peripheiam, cuius diameter eft $=\mathbf{I}$. Summa autem huius feriei $a+b+\frac{3}{4}+$ etc. pendet a fumma ferioi $x+\frac{1}{1}+\frac{1}{9}+\frac{1}{6 e}+\frac{1}{45}+$ etc. quia baec quarta fui parte minuta illam dat. Eft ergo fumma huius feriei acqualis fummae illius cum fui triente. Quamobrem erit $\mathrm{x}+\frac{1}{4}+\frac{1}{0}+\frac{1}{85}+\frac{1}{95}+\frac{1}{5 s}-1$-etc. $=p^{2}$, ideoque buins feriei fumma per 6 multiplicata aequalis eft quadrato peripheriae circuli cuius diameter eft $\mathbf{I}$; quae eft ipfa propofitio cuius initio mentionem feci.

§. 12. Cum igitur cafu quo $y=\mathrm{x}$, fit $\mathrm{P}=\mathrm{x}$ et $\mathrm{Q}=\mathrm{x}$, crunt reliquarum litterarum $\mathrm{R}, \mathrm{S}, \mathrm{T}, \mathrm{V}$ etc. vt fequitur: $\mathrm{R}=\frac{1}{2} ; \mathrm{S}=\frac{1}{3} ; \mathrm{T}=\frac{3}{4} ; \mathrm{V}=\frac{2}{15} ; \mathrm{W}=\frac{6 \mathrm{r}}{72}$; $\mathrm{X}={ }_{\mathrm{r}}$ etc. Cum autem fumma cuborum ipfi $\mathrm{R}=\frac{1}{4}$ fit aequalis, erit $\frac{a^{3}}{a^{3}}\left(\mathbf{x}-\frac{1}{7^{3}}+\frac{1}{3^{3}}-\frac{1}{7^{3}}+\frac{1}{9^{3}}-\right.$ etc. $)=\frac{1}{3}$. Quare erit $x-\frac{1}{7^{3}}+\frac{1}{s^{3}}-\frac{1}{7^{3}}+\frac{1}{v^{3}}-$ etc. $=\frac{q_{4}^{3}}{4}=\frac{p^{3}}{3}$. Huius ideo feriei fumma per 32 multiplicata dat cubum peripheriae circuli cuius diameter eft $x$. Simili modo fumma biquadratorum, quae eft $\frac{2}{p^{2}}\left(x+\frac{1}{3^{4}}+\frac{1}{5^{4}}+\frac{1}{7^{4}}+\frac{1}{3^{4}}+\right.$ etc.) aequalis effe debet $\frac{1}{3}$, ideoque erit $1+\frac{1}{6}+\frac{1}{5^{4}}+$ $\frac{1}{7^{4}}+\frac{1}{9^{4}}+$ etc. $=\frac{q^{4}}{v^{4}}=\frac{p_{6}^{*}}{\text {. }}$. Eft vero haec feries per it multiplicata aequalis huic $\mathrm{I}+\frac{1}{7^{4}}+\frac{7}{3^{4}}+\frac{1}{5^{4}}+\frac{1}{6^{4}}+$ etc. quare ifta feries aequalis eft $p_{0}^{*}$; feu ferici $\bar{x}+\frac{1}{2^{4}}+\frac{1}{3}$ $+\frac{1}{\tau^{*}}+$ etc. fumma per 90 multiplicata dat biquadratum peripheriae circuli cuius diameter eft $\mathbf{I}$.

6. $x_{3}$. Simili modo inuenientur fummae fuperiorum poteftatum; prodibit autem vt fequitur $1-\frac{1}{3}+\frac{1}{5^{3}}$

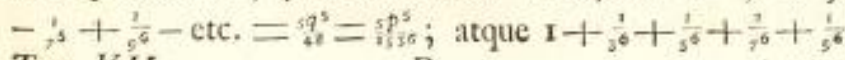
Tom. VII. $\mathbf{R}$ $+\mathrm{ctc}$ 


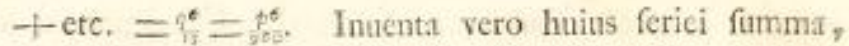
cognofcetur fimul fumma huius feriei $\mathrm{I}+\frac{1}{2}+\frac{1}{6}+\frac{1}{6}$ $+\frac{1}{6}+$ etc. quae crit $=\frac{p^{6}}{4+1}$. Porro pro poteftatibus

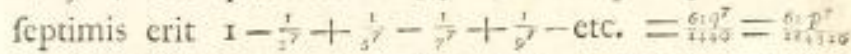
ac pro octaurs $\mathrm{I}-1+\frac{1}{1}+\frac{1}{3^{8}}+\frac{1}{7^{2}}+\frac{\mathrm{T}}{9^{8}}+\mathrm{ctc} .={ }^{177^{2}}=$ xhas $=p^{*}$. Obferuandam autem eft de his feriebus in potentiis exponentum imparium figna terminorum alternari, pro poteftatibus paribus vero effe aequalia; hocque in caufa eft, quod huius generalis feriei $x+\frac{t}{1^{3}}+\frac{t}{x^{2}}+\frac{x}{4}$ + etc. is tantum cafibus fumma polfit exhiberi, quibus $n$ eft numerus par. Practerca quoque notandum eft, fi feriei $\mathrm{I}, \mathrm{X}, \frac{1}{3}, \frac{1}{3}, \frac{5}{4}, \frac{1}{15}, \frac{6}{15}, \frac{1}{14}$ etc. quos valores pro litteris $\mathrm{P}, \mathrm{Q}, \mathrm{R}, \mathrm{S}$ etc. inuenimus, terminus generalis poffet affignari, tum eo ipfo quadraturam circuli cxhibitum iri.

6. 14. In his pofuimus finum PM aequalem ratdio, videamus ergo quales feries prodeant, fi ipfi $y$ alii valores tribuantur. Sit igitur $y=\frac{1}{\sqrt{2}}$, cui finui minimus arcus refpondens eft $\frac{1}{q} p$. Pofito ergo $\mathrm{A}=\frac{1 p}{p}$ crit feries terminorum fimplicium fou primae poteftatis ifla $\frac{t}{p}+$ $\frac{t}{s p}-\frac{t}{s p}-\frac{t}{p p}+\frac{4}{p p}+\frac{\dot{p}}{i p}-$ etc. cuius feriei fumma $\mathrm{P}$ aequalis eft $\frac{i}{y}=V_{2}$. Habebitur ergo $\frac{p}{2 \sqrt{2}}=\mathrm{I}+\frac{1}{y}-\frac{1}{3}-\frac{1}{y}-\frac{1}{y}-\frac{1}{i t}$ $\frac{x}{y}-\frac{t}{2}$ etc. quae feries tantum ratione fignorum a $L e i b-$ nitiana differt, et a Newtono iam dudum eft prohta. Summa vero quadratorum illorum terminorum nempe $\frac{16}{p=}$ $\left(\mathrm{I}+\frac{1}{5}+\frac{1}{y_{j}}+\frac{t}{4}+\right.$-etc. $)$ aequalis eft ipfi $\mathrm{Q}=2$. Frit

ergo 


\section{SERIERIM RECIPROCARTM.}

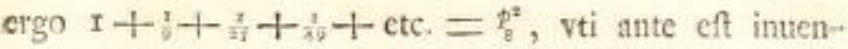
tum.

6. 3. Si flat $y=v_{y}$ erit minimus arcus huic finui refpondens $60^{\circ}$, idcoque $A=\frac{1}{3} p$. Hoc exgo cafu fequens prodibit feries terminorum $\frac{\pi}{p}+\frac{3}{2 p}-\frac{1}{1+p}-\frac{3}{5 p}+\frac{2}{7 p}$ 十- $\frac{T}{p}$ etc. quorum terminorum fumma aegualis eft ipfi

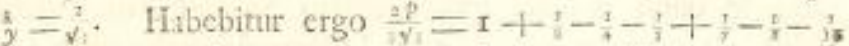
- is + etc. Summa vero quadratorum illorum terminorum eft $=\frac{1}{y^{2}}=\frac{1}{\mathrm{t}}$; vnde fequitur fore $\mathrm{p}^{2}=\mathrm{I}+\frac{1}{+}+$ $\frac{1}{x}+\frac{1}{3}+\frac{1}{15}+\frac{1}{15}+c t c$. in qua ferie defunt termini ternario conftuntes. Pendet autem haec ferics quoque $a b$ ifta $I+\frac{1}{1}+\frac{1}{2}+\frac{1}{5}$ etc. cuins fumma crat inuenta $=p_{0}^{2}$; nam fi haec ferics fui parte nona minuatux prodit ipfa fiuperior feries, cuius ideo fumma debet effe $\sum_{\sigma}^{2}\left(\mathrm{I}-\frac{1}{b}\right)$ $=$ P. Simili modo fi alii affumantur finus, aliae prodibunt ferics, tam fimplicium, quam terminorum quadestorum altiorumgue poreitatum, quarum fummae quadraturam circuli inuoluent.

6. I6. At fi ponatur $y=0$, huiusmodi feries non amplius affignati poterunt, propter $y$ in denominatorem pofitum, feu aequationem initialem per $y$ diuifam. Alio autem modo feries inde deduci poterunt, guae cum fint ipfae leries $x+\frac{1}{n^{4}}+\frac{1}{n^{3}}+\frac{1}{4^{n}}+$ etc. fi $n$ eft numerus par: quemadmodum harum ferierum fummae fint inecniendae, feorfun ex hoc cafu quo $y=0$ deducam. Pofito vero $y=0$ ipfia acguntio fundamentalis abit in hane

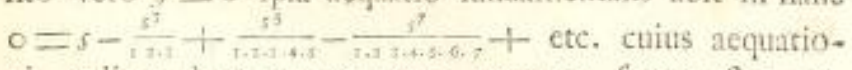
nis radices dant omnes arcus, quorum finus eft $=0$.

$$
\mathrm{R}=
$$

Eit 
Eft autem vna minimaque radix $s=0$, quare aequat $s$ per $s$ diuifa exhibebit reliquos arcus omnes, quorum finus eft $=0$, qui arcus proinde erunt radices huius ac-

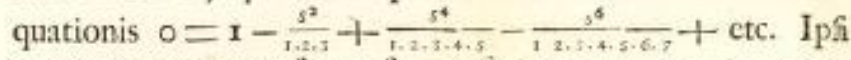
vero arcus quorum finus eft $=0$ lunt $p,-p,+2 p,-2 p$, $3 p,-3 p$ etc. quorum binorum alter alterius eft negatiuus, id quod quoque ipfa aequatio propter dimentiones ipfius $s$ tantum pares indicat. Quare diuifores illius acquationis erunt $\mathbf{x}-\frac{s}{p}, \mathbf{I}+\frac{s}{p}, \mathbf{I}-\frac{s}{2 p}, \mathbf{x}+\frac{s}{2 p}$, etc. atque

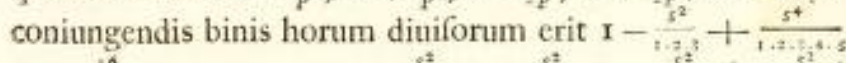
$-\frac{10}{1.5 .5 .55 .7}+$ etc. $=\left(1-\frac{s^{2}}{p^{2}}\right)\left(1-\frac{s^{2}}{4 p^{2}}\right)\left(1-\frac{s^{2}}{2 p^{2}}\right)\left(1-\frac{s^{2}}{16 p^{2}}\right)$ etc.

6. 17. Manifeftum iam eft ex natura aequationum, fore coefficientem ipfius ss feu $\frac{t}{t: 2 s}$ aequalem $\frac{z}{p^{2}}+\frac{t}{4 p^{3}}$ $+\frac{1}{g p^{2}}+\frac{1}{20 p^{2}}+$ etc. Summa vero factorum ex binis terminis huius feriei erit $=\frac{1}{1: \ldots+.+5}$; fummaque factorum ex ternis $=\frac{1}{1,2 \cdot+\cdot+5,5.67} \mathrm{ctc}$. Hanc ob rem erit iuxta 6. $8 . \alpha=\frac{1}{1,2.5} ; b=\frac{1}{1.2 .4 .4 .5} ; \gamma=\frac{1}{1.2 .5 .4 .5 \cdot 6.7} ;$ etc. atque pofita quoque fumma terminorum $\frac{1}{p^{2}}+\frac{1}{4 p^{2}}+\frac{1}{9 p^{2}}+\frac{t}{i \sigma p^{2}}$ + etc. $=\mathrm{P}$, et fumma quadratorum corundem terminorum $=\mathrm{Q}$; fumma cuborum $=\mathrm{R}$; fumma biguacratorum $=\mathrm{S}$; etc. crit per \&. 8. $\mathrm{P}=a=\frac{1}{1,3}=\frac{1}{6} ; \mathrm{Q}=$ $\mathrm{P} \alpha-2 \mathrm{~B}=\frac{1}{30} ; \mathrm{R}=\mathrm{Q} \alpha-\mathrm{Pb}+3 \gamma=\frac{1}{345} ; \mathrm{S}=\mathrm{R} \alpha-\mathrm{Q} b$ $+\mathrm{P} \gamma-4 \delta={ }_{0 * 3 \delta} ; \mathrm{T}=\mathrm{S} a-\mathrm{R} b+\mathrm{Q} \gamma-\mathrm{P} \delta+5 \varepsilon=$

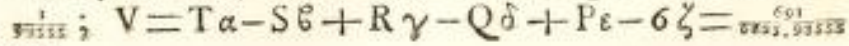
etc.

6. 18. 
5. 18. Ex his ergo deriuantur fummae fequentes:

$$
\begin{aligned}
& \mathrm{I}+\frac{t}{2^{2}}+\frac{t}{\frac{1}{2}^{2}}+\frac{t}{t^{2}}+\frac{1}{s^{2}} \text { etc. }=\frac{p^{2}}{6}=\mathrm{P} \\
& \mathrm{x}+\frac{1}{2^{4}}+\frac{1}{x^{4}}+\frac{1}{4^{4}}+{ }_{5^{4}} \text { etc. }=p_{5}^{4}=\mathrm{Q}
\end{aligned}
$$

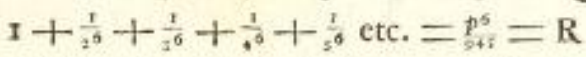

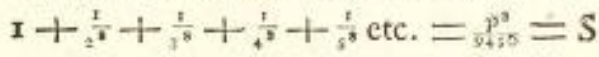

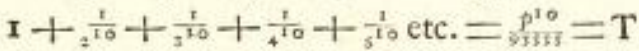

$$
\begin{aligned}
& \mathbf{x}+\frac{1}{3^{12}}+\frac{1}{3^{12}}+\frac{1}{4^{12}}+\frac{1}{5^{12}} \text { etc. }=\frac{69 p^{p 2}}{6825.95555}=\mathrm{V} \text {. }
\end{aligned}
$$

quae feries ex data lege attamen multo labore ad altiorcs poteftates produci poffunt. Diuidendis autem fingulis feriebus per praecedentes orientur fequentes aequa-

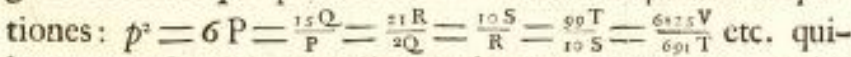
bus expreffionibus fingulis quadratum peripheriae cuius diameter eft $\mathbf{I}$, acquatur.

6. 19. Cum attem harum ferierum fummae etiamf vero proxime facile exhiberi poffent, tamen non multum adiumenti afferre queant ad peripheriam circuli vero proxime exprimendam propter radicem quadratam, quae extrahi deberet; ex prioribus feriebus eliciemus exprefliones, quae ipfi peripheriae $p$ fint acquales. Prodibit autem vt fequitur:

$\mathbf{R}_{3}$

$p=4$

RBHM, Vol. 21, n 42, pp. 206-228, 2021 
334 DE SIMMIS SERIERVM RECIPROC ARVM.

$$
\begin{aligned}
& p=4 .\left(\mathrm{x}-\frac{1}{3}+\frac{1}{2}-\frac{1}{4}+\frac{1}{2}-\frac{\pi}{\pi}+\text { etc. }\right)
\end{aligned}
$$

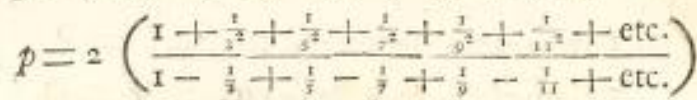

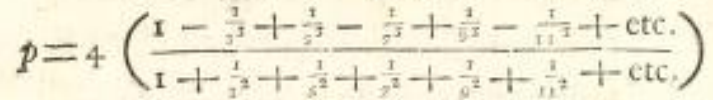

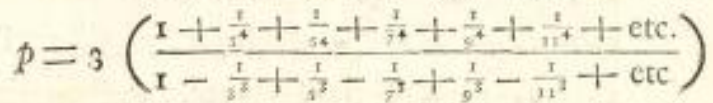

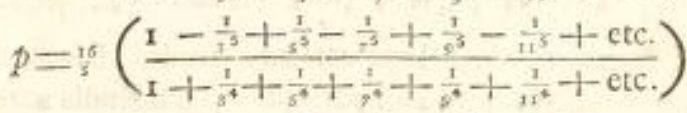

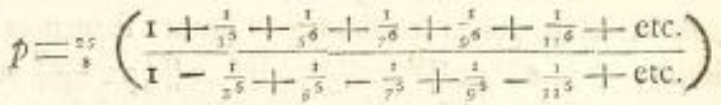

$$
\begin{aligned}
& p=\ell_{1}^{2}\left(\frac{1-\frac{1}{3}+\frac{1}{3}-\frac{1}{7}+\frac{1}{9^{2}}-\frac{1}{11^{7}}+\text { etc. }}{I+\frac{1}{3^{6}}+\frac{1}{5^{6}}+\frac{1}{9^{6}}+\frac{1}{9^{6}}+\frac{1}{11^{6}}+\text { etc. }}\right)
\end{aligned}
$$

DE 